\title{
A quantitative model of the magnetosphere with poloidal vector fields
}

\author{
J. C. Kosik \\ Division Mathematiques, bpi 1244, CNES, 31401 Toulouse Cedex 4, France \\ e-mail: jean-claude.kosik@cnes.fr
}

Received: 12 December 1997 / Revised: 27 April 1998 / Accepted: 15 May 1998

\begin{abstract}
A quantitative model of the magnetospheric magnetic field is developed using poloidal vector fields. This formalism is applied to the ring current region, the distant field and the return currents. The tail model is similar to the unwarped model of Tsyganenko. Several sets of coefficients are obtained for different Kp through a fit of the NSSDC data base. Experimental $\Delta B$ contours and theoretical distributed currents contours are correctly described and are Kp-dependent. Field line topology problems and poor ring current description observed in models of similar complexity are avoided. Computer time has been kept reasonable and makes this model particularly adapted to intensive-type calculations.
\end{abstract}

Key words. Magnetospheric physics (magnetospheric configuration and dynamics).

\section{Introduction}

In the last two decades several magnetic field models have been developed, using different mathematical approaches and with different goals. The models of Alekseev and Shabansky (1972), Voigt (1972), Hilmer and Voigt (1995), Stern (1985) or Schulz and McNab (1996) used a prescribed magnetopause, parabolic, semispherical or more realistic. Some models had a selfconsistent calculated magnetopause, (Olson and Pfitzer, 1974; Choe and Beard, 1974). Only a few of them were based on magnetic field data (Mead and Fairfield, 1975; Tsyganenko and Usmanov, 1982; Tsyganenko, 1987, 1989) or tentatively described the magnetic field topology in some regions of the magnetosphere (Olson and Pfitzer, 1974; Kosik, 1989). This spread in the modelling approaches arose from the difficulty in describing the distant regions or the local deformations created by currents (field aligned currents, ring current). These approaches are in fact complementary, the quantitative models showing their weaknesses when associated to the data, and the theoretical models bringing to light possible mathematical tools. In this respect the works of Schulz and McNab (1987) and Tsyganenko (1996) are particularly instructive. The present study is another example of an old technique used for the modelling of the Earth's dynamo, brought to light by Stern (1976).

The magnetic field is described by a sum of poloidal vector fields and the magnetic field is therefore divergence-free per construction. The ring current region is described by three poloidal functions (Kosik, 1989). The more distant field uses a vector spherical harmonics expansion and the tail model is the 1982 tail model of Tsyganenko and Usmanov (1982). The return currents along the magnetopause are modelled by two axisymmetric cylindrical vector fields. In these equations the coefficients of the distant field are adjusted through a least squares fit of the NSSDC magnetic field data base developed by Fairfield et al. (1994). The coefficients which describe the ring current region are chosen to approximate the $\Delta B$ contours of Sugiura and Poros (1973) and depend on the Kp index. Modelling with a data base through a least squares fit of the data is always a difficult task when past results are examined. Quite often the ring current region was inadequately described and several models exhibited incomplete dayside shielding which produced dayside field line escape. Night side flaring of the field lines is quite often observed. As a consequence a shielding procedure like that developed by Schulz and McNab (1987, 1996) appears almost necessary. In our model we finally adjust a few coefficients in order to obtain perfect shielding in the dayside and approach the Sibeck magnetopause. This method is rather cumbersome but gives interesting results despite a relatively simple model.

\section{The model}

Our model is constructed with poloidal and toroidal vector fields and the magnetic field can be expressed as a 
sum of two terms, the toroidal part $T$ and the poloidal part $P$ (Stern, 1976):

$B=\nabla \times T+\nabla \times \nabla \times P$

This equation can be expressed in cartesian, spherical or cylindrical coordinate systems. In this study we will use only poloidal vector fields which generate toroidal currents (Wolf-Gladrow, 1987). We apply the poloidal vector field description to the ring current region, to the magnetic field of the distant regions and to the return currents along the magnetopause. For sake of simplicity the magnetic field of the tail is the tail model of Tsyganenko (1982).

\subsection{Description of the ring current magnetic field}

The poloidal vector field which describes the ring current is derived from the general equation expressed in spherical coordinates (Kosik, 1989):

$B=\nabla \times\left(\Psi_{1} r\right)+\nabla \times \nabla \times\left(\Psi_{2} r\right)$

where $\Psi_{1}$ and $\Psi_{2}$ are the toroidal and poloidal generating functions and $r$ is the radius vector. From this expansion we retain only the poloidal vector term $\Psi_{2}$ which is expanded in spherical harmonics:

$\Psi_{2}=\sum_{n=1}^{N} \sum_{m=1}^{n} S_{n}(r) P_{n}^{m}(\theta)\left(c_{n m} \cos m \phi+d_{n m} \sin m \phi\right)$

where $S_{n}(r)$ is a general scalar function of $r$, the generating function and the $P_{n}^{m}(\theta)$ are the associated Legendre polynomials. This equation is expressed in solar magnetic coordinates, $\theta, \phi$ are respectively the colatitude and the longitude. Substituting Eq. (3) into Eq. (2) we obtain the three components of $B$ for the 0 -tilt condition:

$$
\begin{aligned}
B_{r}= & \sum_{n=1}^{N} \sum_{m=0}^{n} \frac{S_{n}(r)}{r} n(n+1) P_{n}^{m}(\theta) \\
& \left(c_{n m} \cos m \phi+d_{n m} \sin m \phi\right) \\
B_{\theta}= & \sum_{n=1}^{N} \sum_{m=0}^{n}\left(\frac{S_{n}(r)}{r}+\frac{\partial S_{n}(r)}{\partial r}\right) \\
& \times \frac{\partial P_{n}^{m}(\theta)}{\partial \theta}\left(c_{n m} \cos m \phi+d_{n m} \sin m \phi\right) \\
B_{\varphi}= & \sum_{n=1}^{N} \sum_{m=0}^{n} m\left(\frac{S_{n}(r)}{r}+\frac{\partial S_{n}(r)}{\partial r}\right) \\
& \times \frac{P_{n}^{m}(\theta)}{\sin \theta}\left(d_{n m} \cos m \phi-c_{n m} \sin m \phi\right)
\end{aligned}
$$

The ring current region is characterized by negative $\Delta B$ contours (Sugiura and Poros, 1973). The $\Delta B$ is the scalar difference between the total magnetic field measured by the spacecraft and the magnetic field of internal origin given by a model like IGRF:

$\Delta B=B_{T}-B_{I}$ where $B_{T}$ is the total field measured by the spacecraft and $B_{I}$ is the IGRF magnetic field. For the ring current region a satisfactory description was obtained with two scalar functions for 0-tilt conditions (Kosik, 1989):

$$
\begin{aligned}
B_{r}= & 2 c_{10} \frac{S_{1}(r)}{r} \cos \theta \\
& +6 c_{21} \frac{S_{2}(r)}{r} \cos \theta \sin \theta \cos \phi \\
B_{\theta}= & -c_{10}\left(\frac{S_{1}(r)}{r}+\frac{\partial S_{1}(r)}{\partial r}\right) \sin \theta \\
& +\sqrt{3} c_{21}\left(\frac{S_{2}(r)}{r}+\frac{\partial S_{2}(r)}{\partial r}\right) \cos 2 \theta \cos \phi \\
B_{\phi}= & -\sqrt{3} c_{21}\left(\frac{S_{2}(r)}{r}+\frac{\partial S_{2}(r)}{\partial r}\right) \cos \theta \sin \phi
\end{aligned}
$$

where $S_{1}(r)=r^{3} \exp \left(-k_{1} r^{2}\right)$ and $S_{2}(r)=r^{3} \exp \left(-k_{2} r^{2}\right)$ give a good description of the near-Earth distortion of the magnetic field through the combination of a monomial and an exponential. To take into account the tilt effects, three tilt components must be added:

$$
\begin{aligned}
B_{r}= & \sum_{n=1}^{N} \sum_{m=0}^{n} \frac{S_{n}^{T}(r)}{r} n(n+1) P_{n}^{m}(\theta) \\
& \times\left(c_{n m} \cos m \phi+d_{n m} \sin m \phi\right) \sin T \\
B_{\theta}= & \sum_{n=1}^{N} \sum_{m=0}^{n}\left(\frac{S_{n}^{T}(r)}{r}+\frac{\partial S_{n}^{T}(r)}{\partial r}\right) \frac{\partial P_{n}^{m}(\theta)}{\partial \theta} \\
& \times\left(c_{n m} \cos m \phi+d_{n m} \sin m \phi\right) \sin T \\
B_{\varphi}= & \sum_{n=1}^{N} \sum_{m=0}^{n} m\left(\frac{S_{n}^{T}(r)}{r}+\frac{\partial S_{n}^{T}(r)}{\partial r}\right) \frac{P_{n}^{m}(\theta)}{\sin \theta} \\
& \times\left(d_{n m} \cos m \phi-c_{n m} \sin m \phi\right) \sin T
\end{aligned}
$$

We retain only one generating function and obtain:

$$
\begin{aligned}
& B_{r}^{T}=c_{20} \frac{S_{2}^{T}}{r}\left(9 \cos ^{2} \theta-3\right) \sin T \\
& B_{\theta}^{T}=-c_{20}\left(\frac{S_{2}^{T}}{r}+\frac{\partial S_{2}^{T}}{\partial r}\right) 3 \sin \theta \cos \theta \sin T \\
& B_{\phi}^{T}=0
\end{aligned}
$$

where $S_{2}^{T}=r^{3} \exp \left(-k_{2}^{T} r^{2}\right)$ and $T$ is the tilt angle. The coefficients $c_{10}=-1.5, c_{21}=0.11, k_{1}=0.04, k_{2}=0.01$, $k_{2}^{T}=0.005$ were chosen in order to reproduce $\Delta B$ contours. The coefficient $c_{20}$ can be adjusted. The magnetic field for the ring current is thus the sum of eqs. (6) and (8).

\subsection{The magnetic field of the distant regions of the magnetosphere}

For the magnetic field contribution of the distant regions we use also the spherical harmonics series (Eq. $4 \mathrm{a}, \mathrm{b}, \mathrm{c})$ but in this case functions $S_{n}(r)$ are mono- 
mials in $r^{n}$. In the solar magnetospheric coordinate system the components of the magnetic field are expressed as:

$$
\begin{aligned}
B_{r}= & \sum_{n=1}^{N} \sum_{m=0}^{n} n(n+1)\left(\frac{r}{r_{b}}\right)^{n-1} \\
& \times P_{n}^{m}(\theta)\left\{\left(a_{n m} \cos m \phi+b_{n m} \sin m \phi\right) \cos T\right. \\
& \left.+\left(a_{n m}^{*} \cos m \phi+b_{n m}^{*} \sin m \phi\right) \sin T\right\} \\
B_{\theta}= & \sum_{n=1}^{N} \sum_{m=0}^{n}(n+1)\left(\frac{r}{r_{b}}\right)^{n-1} \\
& \times \frac{\partial P_{n}^{m}(\theta)}{\partial \theta}\left\{\left(a_{n m} \cos m \phi+b_{n m} \sin m \phi\right) \cos T\right. \\
& \left.+\left(a_{n m}^{*} \cos m \phi+b_{n m}^{*} \sin m \phi\right) \sin T\right\} \\
B_{\phi}= & \sum_{n=1}^{N} \sum_{m=0}^{n}(n+1)\left(\frac{r}{r_{b}}\right)^{n-1} \frac{m}{\sin \theta} \\
& \times P_{n}^{m}(\theta)\left\{\left(b_{n m} \cos m \phi-a_{n m} \sin m \phi\right) \cos T\right. \\
& \left.+\left(b_{n m}^{*} \cos m \phi-a_{n m}^{*} \sin m \phi\right) \sin T\right\}
\end{aligned}
$$

where $\theta$ is the colatitude, $\phi$ the longitude and $r$ the radial distance. These equations are similar to the usual spherical harmonics expansions derived from a scalar potential. They differ only by the factors $n+1, \cos T$ and $\sin T$ and powers of $\frac{r}{r_{b}}$ and classical routines developed for inner field calculations can be partially re-used. The three components take into account the amount of tilt and are multiplied by $\cos T$ and $\sin T$. The best results were obtained retaining only terms in $\cos T$. Symmetry conditions should be taken into account. In the case of 0 tilt, when $\phi \rightarrow-\phi$ we have $B_{r} \rightarrow B_{r}, B_{\theta} \rightarrow B_{\theta}, B_{\phi} \rightarrow-B_{\phi}$. When the tilt is different from zero for $T \rightarrow-T$ and $\theta \rightarrow \pi-\theta$, we have
$B_{r}(-T) \rightarrow-B_{r}(T), B_{\theta}(-T) \rightarrow B_{\theta}(T), B_{\phi}(-T) \rightarrow-B_{\phi}(T)$. Applying these conditions to expression $(9 \mathrm{a}, \mathrm{b}, \mathrm{c})$ the coefficients $b_{n m}$ vanish. The remaining coefficients $a_{n m}$ multiplied by $\cos T$ vanish when $n+m$ is even. The final formulae for the components of the distant field are:

$$
\begin{aligned}
B_{r}= & \sum_{n=1}^{N} \sum_{m=0}^{n} n(n+1)\left(\frac{r}{r_{b}}\right)^{n-1} \\
& \times P_{n}^{m}(\theta) a_{n m} \cos m \phi \cos T \\
B_{\theta}= & \sum_{n=1}^{N} \sum_{m=0}^{n}(n+1)\left(\frac{r}{r_{b}}\right)^{n-1} \\
& \times \frac{\partial P_{n}^{m}(\theta)}{\partial \theta} a_{n m} \cos m \phi \cos T \\
B_{\phi}= & -\sum_{n=1}^{N} \sum_{m=0}^{n}(n+1)\left(\frac{r}{r_{b}}\right)^{n-1} \\
& \times \frac{m}{\sin \theta} P_{n}^{m}(\theta) a_{n m} \sin m \phi \cos T
\end{aligned}
$$

The coefficients $a_{10}, a_{21}, a_{30}, a_{32}, a_{41}, a_{43}, a_{50}, a_{52}, a_{54}$ are given in Table 1 for different $\mathrm{Kp}$ intervals.

\subsection{The return currents}

The representation of return currents should take into account the cylindrical shape of the tail. Therefore poloidal vector fields expressed in cylindrical coordinates are particularly adapted. The cylindrical coordinate system should follow the tilt of the dipole near the Earth and the upward motion of the neutral sheet. We choose for the solution a sum of poloidal vector fields of the form:

$B_{c}=\sum_{i} \nabla \times \nabla \times\left(\hat{e}_{z} \psi_{i}\right)$

where the unit vector $\hat{e}_{z}$ is along the axis $x g s m$ in the Sun direction, or parallel to this axis. The poloidal functions have the following form:

Table 1. Various coefficients and their Kp relationships

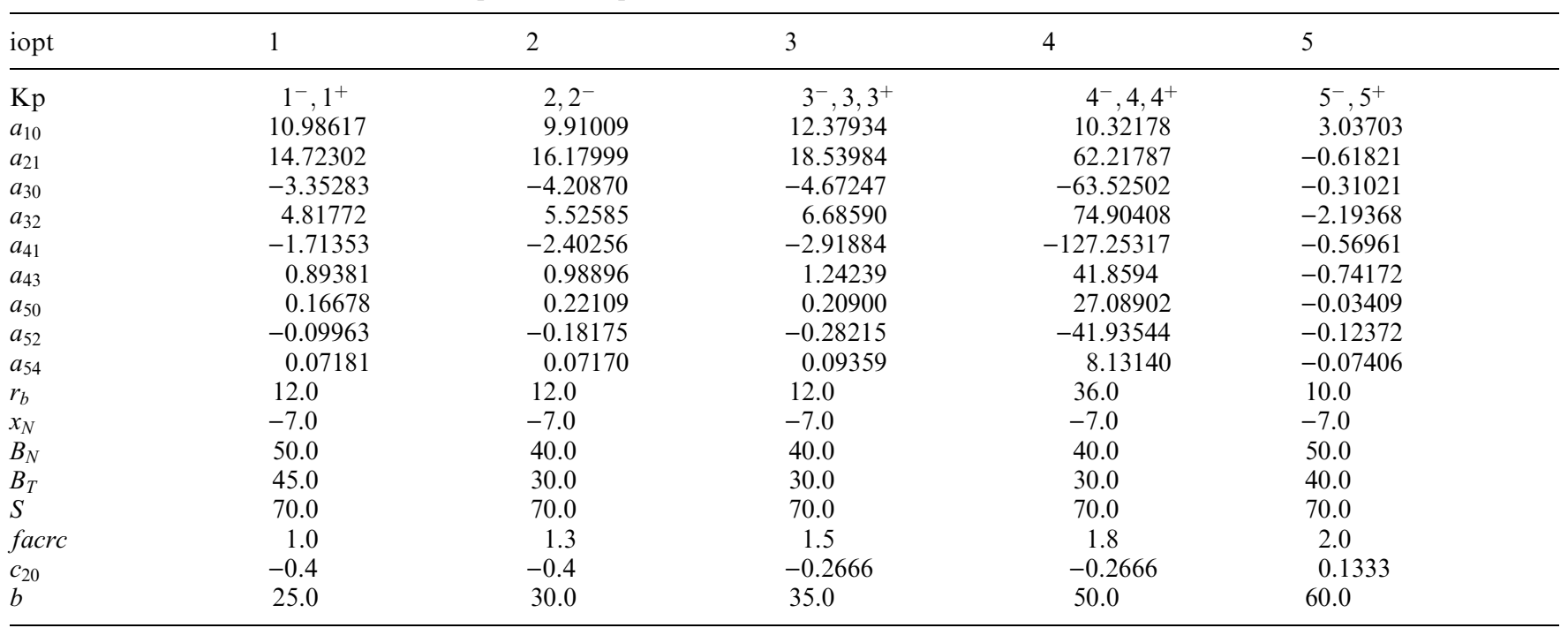


$\psi_{i}=J_{m}(\rho)\left(a_{m i} \cos m \lambda+b_{m i} \sin m \lambda\right) e^{k z}$

where $J_{m}(\rho)$ is the Bessel function of order $m$ and $\lambda$ is counted from the ygsm axis (solar magnetospheric coordinate system) and $\rho$ is counted from the associated $x$ axis. The solution will contain vector fields linked to the upward motion of the neutral sheet and vector fields associated to the tilt of the dipole. The characteristic radius of the Bessel functions, $R_{o}$, is chosen in accordance with the radius of the tail, $\left(R_{o}=30 \mathrm{Re}\right)$ and the constant $k$ in the exponential is set to 0.05 for all the Bessel expansions. In the following part of the text we will omit the subscript $i$ and the summation sign for the clarity of the mathematical expressions but a general solution can always be obtained as a sum of the elementary solutions with various constants $a_{m i}, b_{m i}$.

We can rewrite Eq. (11) as $B_{c}=\nabla \times B_{1}$ with

$B_{1}=\nabla \times \hat{e}_{z} \psi=\nabla \psi \times \hat{e}_{z}$

we get:

$B_{1 \rho}=m \frac{J_{m}}{\rho}\left\{b_{m} \cos m \lambda-a_{m} \sin m \lambda\right\} e^{k z}$

$B_{1 \varphi}=-\frac{\mathrm{d} J_{m}}{\mathrm{~d} \rho}\left\{a_{m} \cos m \lambda+b_{m} \sin m \lambda\right\} e^{k z}$

$B_{1 z}=0$

Taking the curl of $B_{1}, B_{c}=\nabla \times B_{1}$ we obtain

$B_{c \rho}=k \frac{\mathrm{d} J_{m}}{\mathrm{~d} \rho}\left(a_{m} \cos m \lambda+b_{m} \sin m \lambda\right) e^{k z}$

$B_{c \lambda}=k m \frac{J_{m}}{\rho}\left(b_{m} \cos m \lambda-a_{m} \sin m \lambda\right) e^{k z}$

$B_{c z}=J_{m}\left(a_{m} \cos m \lambda+b_{m} \sin m \lambda\right) e^{k z}$

In the last equation we have taken into account the definition of the Bessel function through its differential equation:

$\rho^{2} \frac{\mathrm{d}^{2} J_{m}}{\mathrm{~d} \rho^{2}}+\rho \frac{\mathrm{d} J_{m}}{\mathrm{~d} \rho}+\left(\rho^{2}-m^{2}\right) J_{m}=0$

We take into account the properties of the Bessel functions:

$2 \frac{m}{\rho} J_{m}=J_{m-1}+J_{m+1}$

$2 \frac{\mathrm{d} J_{m}}{\mathrm{~d} \rho}=J_{m-1}-J_{m+1}$

We rewrite Eq. (15) as:

$B_{c \rho}=\frac{k}{2}\left[J_{m-1}-J_{m+1}\right]\left(a_{m} \cos m \lambda+b_{m} \sin m \lambda\right) e^{k z}$

$B_{c \lambda}=\frac{k}{2}\left[J_{m-1}+J_{m+1}\right]\left(b_{m} \cos m \lambda-a_{m} \sin m \lambda\right) e^{k z}$

$B_{c z}=J_{m}\left\{a_{m} \cos m \lambda+b_{m} \sin m \lambda\right\} e^{k z}$

The currents are obtained by taking the curl of Eq. $(15 a, b, c)$. We obtain:

$$
\begin{aligned}
& j_{c \rho}=m \frac{J_{m}}{\rho}\left(b_{m} \cos m \lambda-a_{m} \sin m \lambda\right)\left[1-k^{2}\right] e^{k z} \\
& j_{c \lambda}=-\frac{\mathrm{d} J_{m}}{\mathrm{~d} \rho}\left(a_{m} \cos m \lambda+b_{m} \sin m \lambda\right)\left[1-k^{2}\right] e^{k z} \\
& j_{c z}=0
\end{aligned}
$$

These equations can be rewritten as:

$$
\begin{aligned}
j_{c \rho}= & \frac{1}{2}\left[J_{m-1}+J_{m+1}\right] \\
& \times\left(b_{m} \cos m \lambda-a_{m} \sin m \lambda\right)\left[1-k^{2}\right] e^{k z} \\
j_{c \lambda}= & -\frac{1}{2}\left[J_{m-1}-J_{m+1}\right] \\
& \times\left(a_{m} \cos m \lambda+b_{m} \sin m \lambda\right)\left[1-k^{2}\right] e^{k z} \\
j_{c z}= & 0
\end{aligned}
$$

For our modelling problem we have used only two such elementary solutions. One solution describes the fields from the subsolar region down to the earthward boundary of the neutral sheet $x_{n}$. This component is subject to the dipole tilt. The other solution describes the fields from the earthward boundary of the neutral sheet $x_{n}$ down to $x g s m=-40 R e$. The tail component will follow the upward or the downward motion of the neutral sheet when the dipole tilts. The tilt and non-tilt components join at $x g s m=x_{n} \times \cos (T)$ where $x_{n}$ is the Earthward boundary of the tail neutral sheet. $T$ is the tilt angle and $x_{n}$ is set to $-7 \mathrm{Re}$.

For 0 tilt the Bessel functions of the two components have the same $x$ axis which coincides with the $x g s m$ axis. When the tilt is different from zero the axis of the tail Bessel functions moves upward and coincides with the neutral sheet, while the axis of the other component is tilted as shown in Fig. 1. Up to this point the form of Eq. (18) and (20) has not been defined. The physics implies an azimuthal flow of the currents in opposite directions (Fig. 1). In Eq. (20) the trigonometric

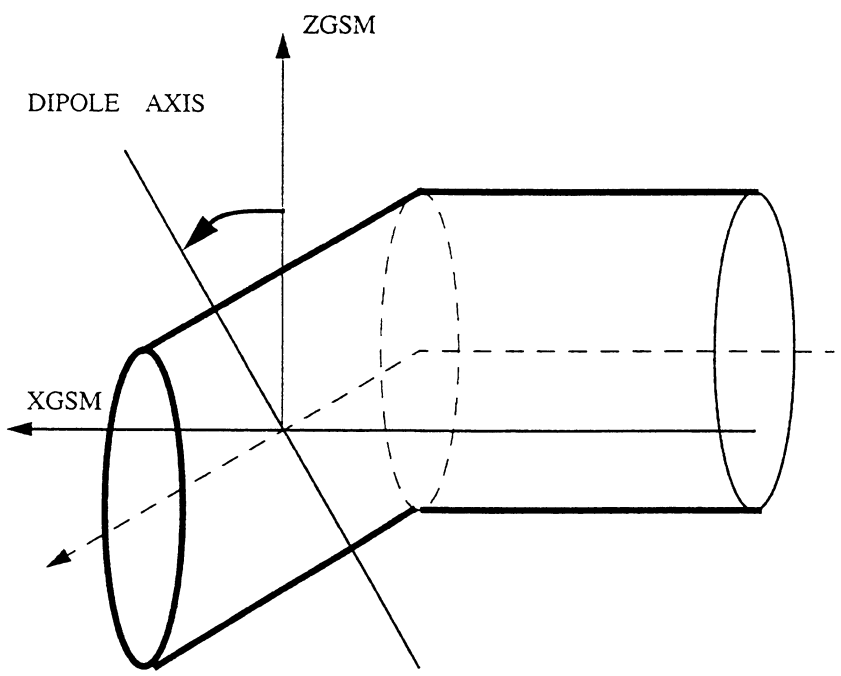

Fig. 1. The return currents can be represented by two poloidal vector fields expressed in a cylindrical coordinate system. These cylindric functions join at the edge of the neutral sheet. One cylinder tilts while the other moves upward. The characteristic radius of the Bessel functions is $30 \mathrm{Re}$ 
expansion should be of the form $\sin (2 n+1) \lambda$. We have $m=2 n+1$ and for $n=0$ the expansion reduces to $\sin \lambda$ The components for the magnetic field are:

$$
\begin{aligned}
& B_{c \rho}=\frac{k}{2}\left(J_{0}-J_{2}\right) b \sin \lambda e^{k z} \\
& B_{c \lambda}=-\frac{k}{2}\left(J_{0}+J_{2}\right) b \cos \lambda e^{k z} \\
& B_{c z}=J_{1} b \sin \lambda e^{k z}
\end{aligned}
$$

For the return currents we have:

$$
\begin{aligned}
& j_{c \rho}=\frac{1}{2}\left(J_{0}-J_{2}\right) b \cos \lambda e^{k z}\left(1-k^{2}\right) \\
& j_{c \lambda}=-\frac{1}{2}\left(J_{0}-J_{2}\right) b \sin \lambda e^{k z}\left(1-k^{2}\right) \\
& j_{c z}=0
\end{aligned}
$$

In our model the subsolar and the tail components have the same form previously defined. For the calculation of the magnetic field or the currents at a given point it is only necessary to take into account the location of the point with respect to the Earthward boundary of the neutral sheet $x_{n}$.

\subsection{The tail field model}

For sake of simplicity we use the model developed by Tsyganenko and Usmanov (1982) which does not have warping. We recall his equations in the gsm coordinate system:

$$
\begin{array}{r}
B_{t x}=\left[\frac { z } { \pi ( z ^ { 2 } + D ^ { 2 } ) ^ { \frac { 1 } { 2 } } } \left(B_{N}\right.\right. \\
\left.-\frac{x_{N}-x}{S} B_{T}\right) F(x, z) . \\
\left.+\frac{B_{T}}{2 \pi S} z G(x, z)\right] f(y)
\end{array}
$$

$$
\begin{aligned}
B_{t y}= & 0 \\
B_{t z}= & {\left[\left(B_{N}-\frac{x_{N}-x}{S} B_{T}\right) \frac{G(x, z)}{2 \pi} .\right.} \\
& \left.+\frac{B_{T}}{\pi}\left(1-\frac{\left(z^{2}+D^{2}\right)^{\frac{1}{2}}}{S} F(x, z)\right)\right] f(y)
\end{aligned}
$$

where

$$
\begin{aligned}
& F(x, z)=\tan ^{-1} \frac{x_{N}-x}{\left(z^{2}+D^{2}\right)^{\frac{1}{2}}}-\tan ^{-1} \frac{x_{N}-x-S}{\left(z^{2}+D^{2}\right)^{\frac{1}{2}}} \\
& G(x, z)=\operatorname{Ln} \frac{\left(x_{N}-x\right)^{2}+z^{2}+D^{2}}{\left(x_{N}-x-S\right)^{2}+z^{2}+D^{2}} \\
& f(y)=\left[1+\left(\frac{y}{\Delta y}\right)^{2}\right]^{-1} \text { and } S=x_{N}-x_{F}
\end{aligned}
$$

In these equations $x_{N}$ and $x_{F}$ are the positions of the inner and outer edges of the current sheet, $D$ is the half thickness of each filament in the tail current sheet. $S$ is the width of the sheet in the $\mathrm{x}$ direction, $f(y)$ is an attenuation function. Typical values are $D=2 R_{e}$,
$\Delta y=10 R_{e}, x_{n}=-7 R_{e} . S, B_{N}, B_{T}$ are the parameters we have chosen to adjust.

When the tilt is different from zero, the neutral sheet moves upwards or downwards and the location of the point of $g s m$ coordinates (xgsm, ygsm, zgsm) is located at a distance $z$ defined as $z=z g s m-\left\|x_{N}\right\| \sin T$ from the neutral sheet where $T$ is the tilt angle.

\section{The use of the database}

A large database (more than 79000 records) has been built up by Fairfield et al. (1994). The magnetic field measurements extend to $-70 \mathrm{Re}$ in the tail, but there is no data inside a sphere of $4 \mathrm{Re}$. Figures 2 and 3 display the whole set of data for all the tilt angles for $\mathrm{Kp}=1$ and $\mathrm{Kp}=5$ respectively in the xgsm,ygsm and xgsm,zgsm planes, where xgsm,ygsm,zgsm are the solar magnetospheric coordinates. Several models have been built using this database or part of this data base (Mead and Fairfield, 1975; Tsyganenko, 1987, 1989). For hightilt angles field line escape in the day side is quite often observed in these models as well as night side field line flaring. This phenomenon is also obtained in our first tentative uses of the database and we have tried to find the origin of this phenomenon. The database was divided in subsets for each $\mathrm{Kp}$ interval and a double filtering was made on both the tilt angle and the location. Data was selected for tilt values in the range $\left(30-35^{\circ}\right)$ and near the magnetopause using the model of Sibeck et al. (1991) within ygsm values between $-5 \mathrm{Re}$ and +5 Re. Results are plotted in Figs. 4 and 5 for $\mathrm{Kp}=1$ and $\mathrm{Kp}=5$ respectively. These figures show clearly the lack of data near the boundary for high-tilt angles and above the dipole poles. This has important consequences as a least squares fit of the data will incorporate almost no information at high latitudes and high-tilt angles indicating the presence of a boundary in these regions and for these tilt conditions. As a consequence the coefficients of the model will not contain this information and field line escape will be observed. The lack of data near the Earth results in a poor description of the ring current region in the absence of an "ad-hoc" ring current model (Mead and Fairfield, 1975). In Tsyganenko (1987, 1989) models the ad-hoc ring current could not describe the eastward and westward components of the ring current. This can be noticed in his Fig. 6 (Tsyganenko, 1989) compared to Fig. 5 (Hilmer and Voigt, 1995).

However the introduction of a better ring current description increases the field line escape: the creation of a negative $\Delta B$ bay in the ring current region counteracts the magnetic field compression in the day side. To avoid this problem theoretical models previously developed used a predefined magnetopause, spherical (Voigt, 1972), parabolic (Alekseev and Shabansky, 1972; Stern, 1985) or with a more realistic shape (Schulz and $\mathrm{McNab}, 1996)$. In these models the following equation should be fulfilled for any point of the magnetopause:

$$
\left(\vec{B}_{d}+\vec{B}_{e}\right) \cdot \hat{n}=0
$$



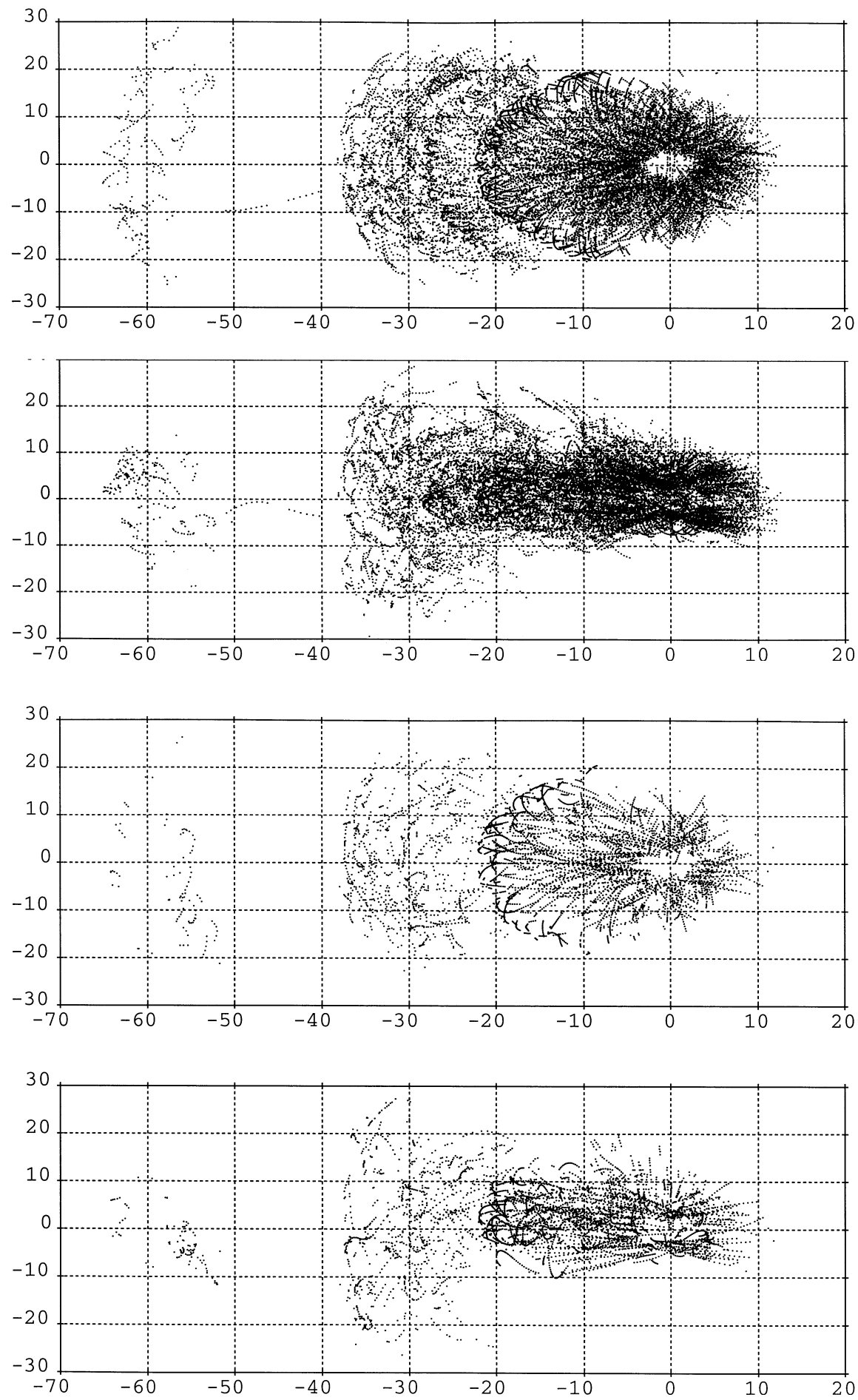

Fig. 2. In these two plots the location of the data points for a low geomagnetic activity level $(\mathrm{Kp}=1)$ are displayed in the solar magnetospheric coordinate system planes xgsm-ygsm and xgsm-zgsm. Notice the absence of points near $x g s m=0$ and for $z g s m$ values greater than $14 \mathrm{Re}$ or below $-10 \mathrm{Re}$. Notice also the lack of points for distances less than $4 \mathrm{Re}$
Fig. 3. Similar to Fig. 2 but for a high geomagnetic activity level $(\mathrm{Kp}=5)$. A lack of measurements is observed over the polar regions where field line escape usually occur where $\vec{B}_{d}$ is the dipole field, $\vec{B}_{e}$ the magnetospheric magnetic field and $\hat{n}$ is the normal to the magnetopause at that point. Since the shape of the magnetopause is given and the dipole field known, the external field inside the magnetosphere must fulfill the stated condition on the boundary. Schulz and McNab (1996), and Tsyganenko (1995) have applied the constraint in a least squares sense:

$\int_{m p}(\hat{n} \cdot B)^{2} \mathrm{~d} S$ where the integral is calculated over the magnetopause surface. In the present work we have adopted a different approach using an iterative process. The NSSDC database is divided into several subsets for different $\mathrm{Kp}$ values from $0,0^{+}$to $5^{-}, 5,5^{+}$. Least squares are performed on these data sets using the tail model with coefficients $B_{T}=10, B_{N}=20, \mathrm{~S}=70$, the ring current contribution is set to zero, as well as the return currents. The subsolar distance is adjusted for the various data subsets. The least squares give the coefficients $a_{n m}$. In the second step the ring current is switched on and its 


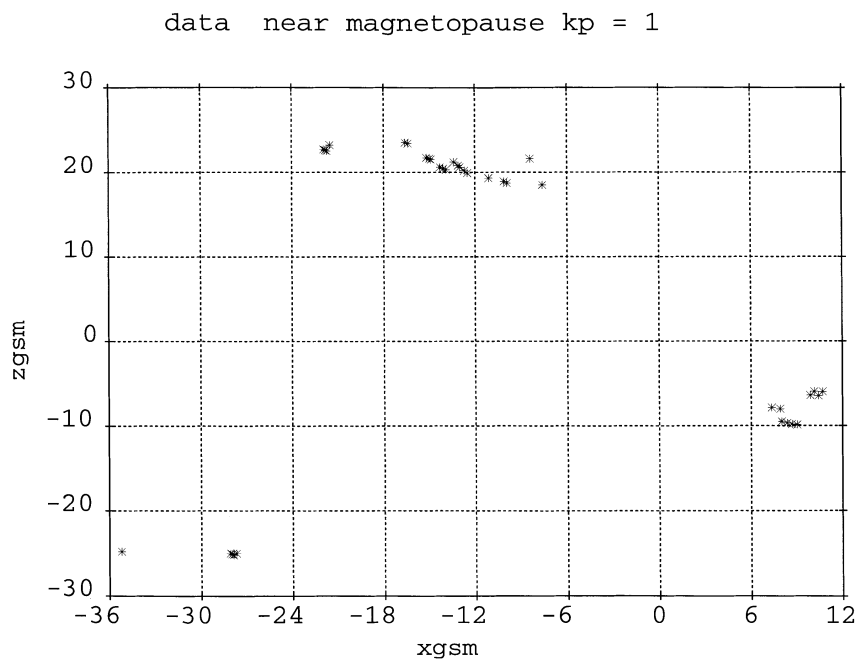

Fig. 4. The data set for $\mathrm{Kp}=1$ has been filtered to retain points located over the polar regions. Retained points have a ygsm coordinate lower than $5 \mathrm{Re}$ and have a zgsm coordinate higher than zmin. zmin is the local zgsm coordinate of the Sibeck magnetopause minus 2 Re. The lack of data along the magnetopause boundary even within a thickness of $2 \mathrm{Re}$ can be noticed

intensity adjusted with a multiplying factor, facrc, in order to approximate the experimental $\Delta B$ contours obtained by Sugiura and Poros (1973). This factor equals 1 for $\mathrm{Kp}=1$ and 2 for $\mathrm{Kp}=5$. At this stage of the work, the tracing of the field lines usually give poor results, with a field line escape in the dayside for high-tilt angles.

In the third step the return currents and the tilt part of the ring current are introduced and the corresponding coefficients $b$ and $c_{20}$ can be adjusted. A careful choice of these two parameters eliminates the field line escape in the dayside for tilt angles up to $35^{\circ}$. Our tentatives indicate that a change as small as 0.01 in one key

data near magnetopause $\mathrm{kp}=5$

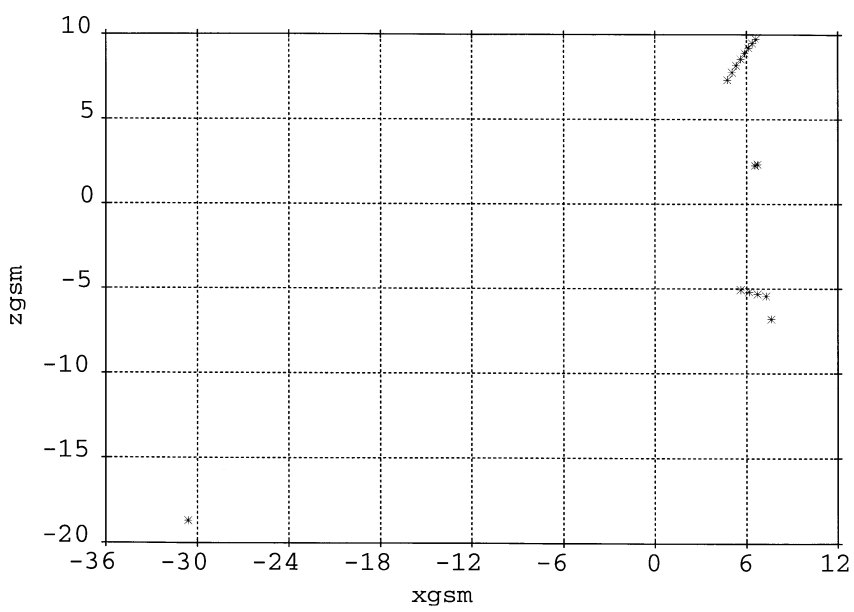

Fig. 5. Same as Fig. 4. The data set for $\mathrm{Kp}=5$ has been filtered in the same conditions with the Sibeck magnetopause model adapted to $\mathrm{Kp}=5$. The lack of data points is even more important

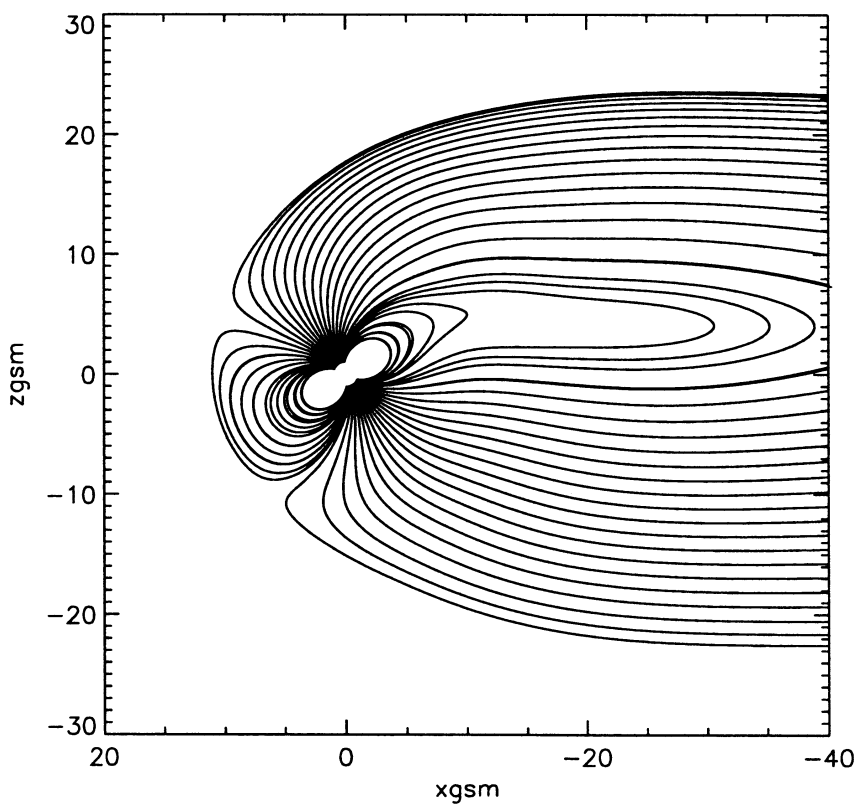

Fig. 6. Field lines are plotted for colatitudes lower than $28^{\circ}$ or higher than $162^{\circ}$ with a $2^{\circ}$ step for $\mathrm{Kp}=1$ and tilt $=35^{\circ}$. The diameter of the tail for $x g s m=-40 \mathrm{Re}$ is approximately $46 \mathrm{Re}$. The subsolar point distance is around $12 \mathrm{Re}$

coefficient induces dayside field line escape. In a final step the tail parameters $B_{N}, B_{T}$ are modified in order to approach the Sibeck magnetopause boundary. In the present model we use only one component of the spherical harmonics expansion, in $\cos T$, with 9 coefficients. Other tentatives with a higher number of

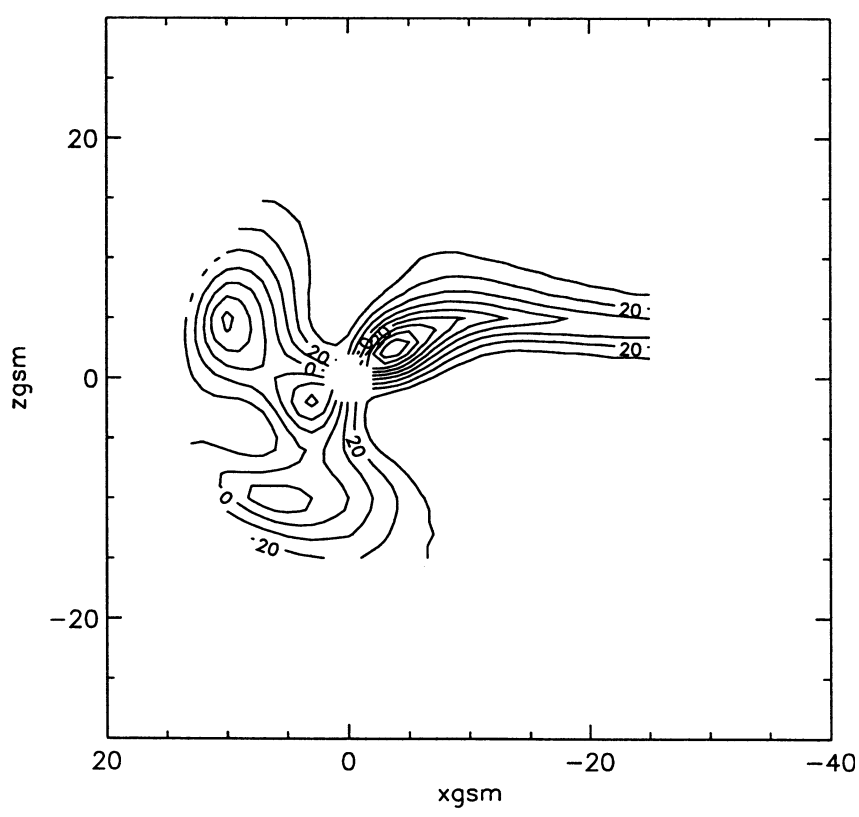

Fig. 7. $\Delta B$ contours are obtained for $\mathrm{Kp}=1$ and tilt $=35^{\circ}$ with a 10 nanoteslas step. In the ring current region the depression is around -30 nanoteslas in the day side and -50 nanoteslas in the night side. Negative bays are obtained near the cusps while positive contours are observed near the subsolar point 


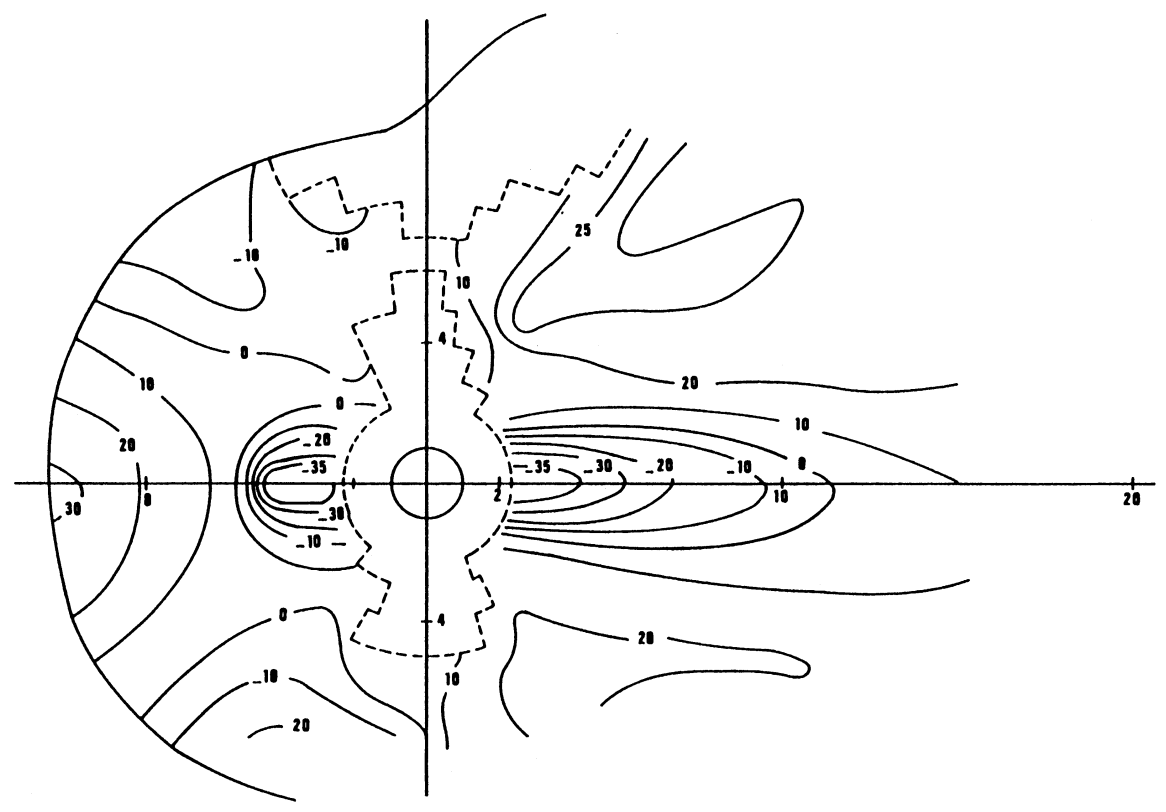

Fig. 8. The experimental $\Delta B$ contours have been plotted for $\mathrm{Kp}=1 . \Delta B$ is the scalar difference between the total field and the internal field of the Earth. These contours are averaged over the tilt. The step between two contours is 10 nanoteslas coefficients gave poor results, with an important uncertainty for the higher order coefficients. The coefficients are given in Table 1 and are discontinuous with $\mathrm{Kp}$ values.

\section{The results}

In Figs. 6 and 7 the magnetic field lines and the $\Delta B$ contours have been plotted for $\mathrm{Kp}=1$ and tilt $=35^{\circ}$. The $\Delta B$ contours are in good agreement with the experimental results of Sugiura and Poros (1973) (Fig. 8). Figures 9 and 10 give the field line topology and the $\Delta B$ contours for $\mathrm{Kp}=5$ and tilt $=35^{\circ} . \Delta B$ contours show higher depressions in the ring current region and field lines are more compressed and the field line escape is avoided. These results are $\mathrm{Kp}$-dependent and should be compared to the corresponding models of Tsyganenko (1987, 1989). However the field line topology and the near-Earth region description make our model similar to the latest model of Tsyganenko 1996_V1 (Tsyganenko, 1996) with the following set of parameters: $\mathrm{imf}=0, \mathrm{dst}=0$, pdyn $=2$ for $\mathrm{Kp}=1$ and $\operatorname{imf}=0$, dst $=20$, pdyn $=6$ for $\mathrm{Kp}=5$. The complexity of our analytical formulation is far less important compared to this last model, even if the field aligned currents are not described. As a consequence the computing time for field lines and $\Delta B$ contours requires $60 \mathrm{~s}$ versus $12 \mathrm{~s}$ for the Tsyganenko 1989 model and 480 seconds for the Tsyganenko 1996_V1 model on a Sun Sparc 5 workstation. We have calculated the distributed currents, $J=\nabla \times B$. The results for the ygsm component are plotted in Figs. 11 and 12 for Kp $=1$ and $\mathrm{Kp}=5$ for a $35^{\circ}$ tilt angle. In these plots positive values correspond to current flow in the direction of the positive ygsm axis and negative values correspond to current flow in the direction of the negative ygsm axis.
The current flow bends with the tilt of the dipole axis in the ring current region. In this region the two components of the flow are present, an eastward flow near the Earth and a westward flow in the outer region and can be compared to the self-consistent results of Sozou and Windle (1969) and the model of Hilmer and Voigt (1995). This feature was absent in the earlier models of Tsyganenko (Fig. 6, 1989). The increase in geomagnetic activity results in an increase of the intensity of the currents. In the tail, positive values indicate the dawn dusk flow of currents in the neutral sheet.

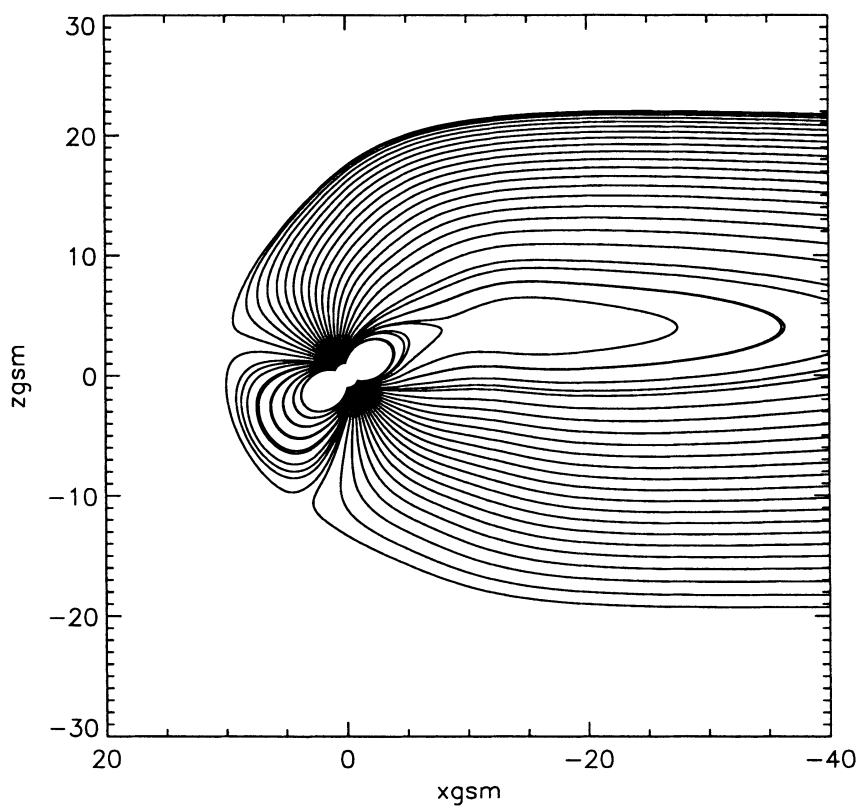

Fig. 9. Same as Fig. 6 but for $\mathrm{Kp}=5$. The field lines are more compressed. The diameter of the tail is around $40 \mathrm{Re}$ and the subsolar point is located at $10 \mathrm{Re}$ 


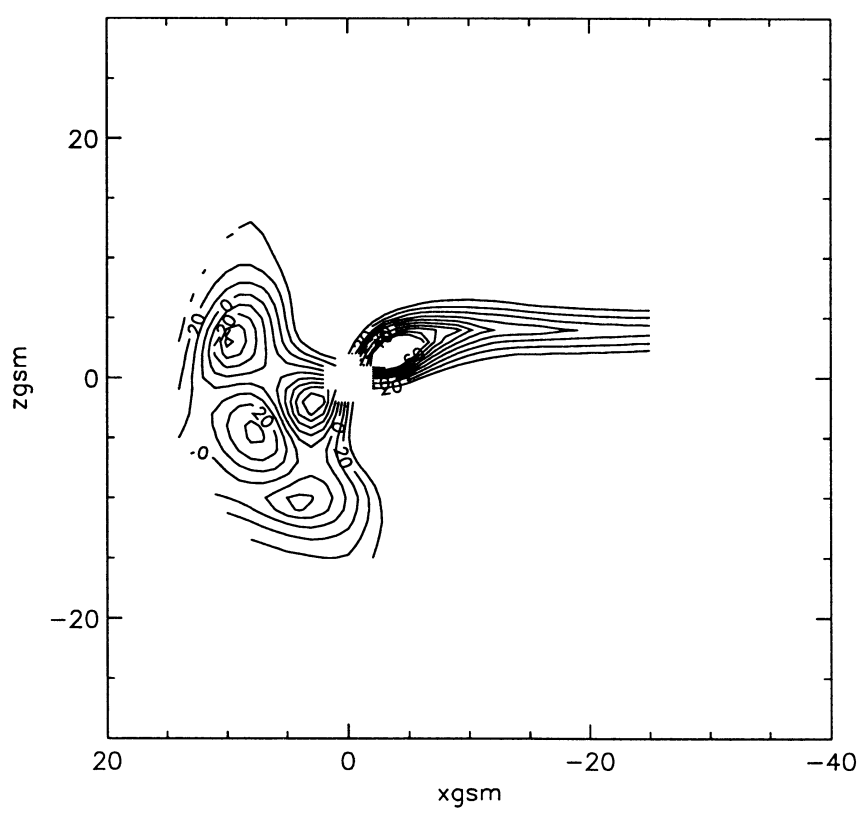

Fig. 10. The $\Delta B$ contours are obtained for $\mathrm{Kp}=5$ and tilt $=35^{\circ}$. The ring current depression reaches -50 nanoteslas in the day side and is lower than -60 nanoteslas in the night side. The step between two contours is 10 nanoteslas

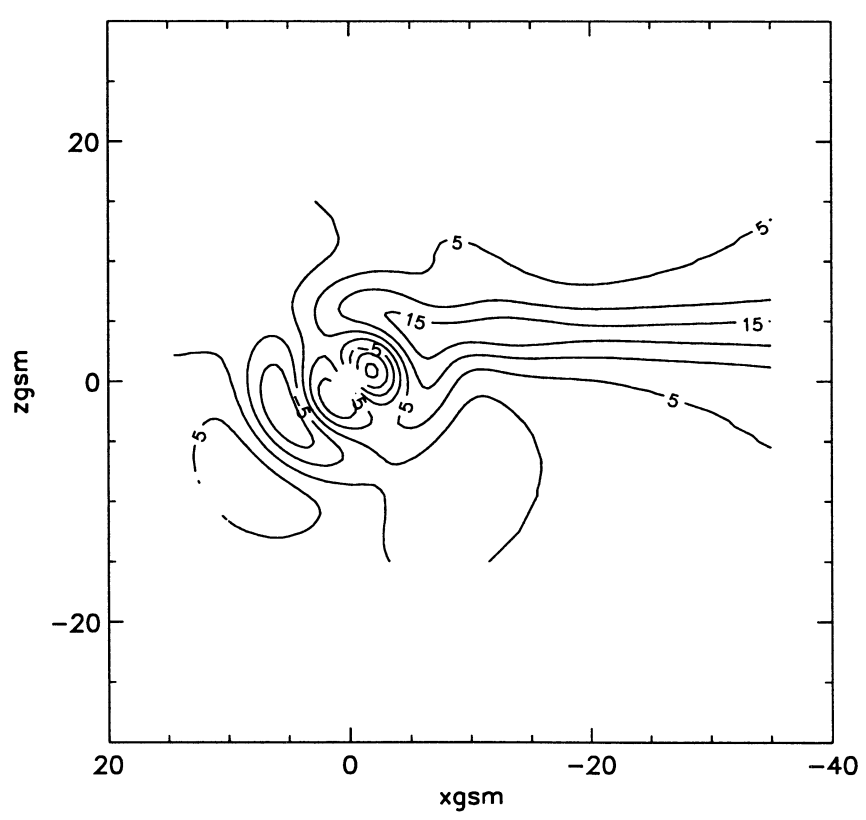

Fig. 11. The $J_{y}$ component of the current density contours is plotted for $\mathrm{Kp}=1$. Positive values correspond to current flow in the direction of the positive ygsm axis. Positive ygsm axis is perpendicular to the $x g s m-z g s m$ plane and points out of the figure. Negative values correspond to current flow in the direction of the negative ygsm axis. There is an eastward flow near the Earth and a westward flow in the outer region. In the night side the westward current merges smoothly with the dawn-dusk current flow of the neutral sheet. Quantities should be multiplied by $1.2 \cdot 10^{-10} \mathrm{~A} / \mathrm{m}^{2}$

\section{Conclusions}

The present results indicate that the poloidal vector field formalism can be applied to the modelling of almost all

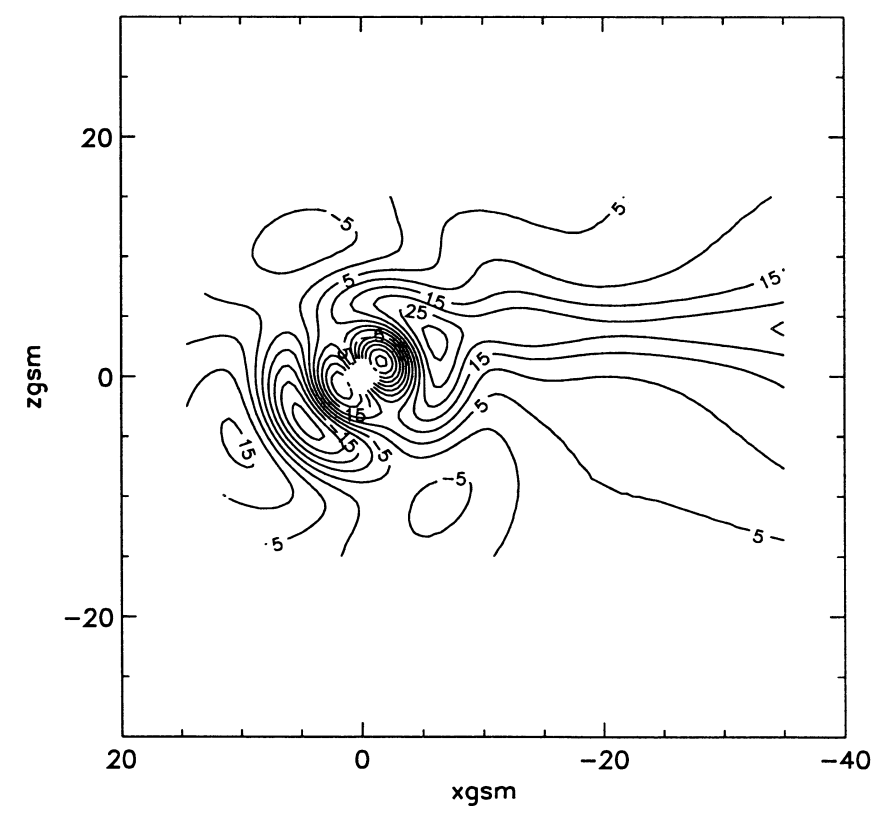

Fig. 12. Same as Fig. 11 but for a geomagnetic activity level $\mathrm{Kp}=5$. Eastward and westward currents are enhanced by a factor two

the regions of the magnetosphere to good effect. The ring current region and the distant field are described with vector spherical harmonics but the generating function is adapted to each particular topology. The ring current region is described by the product of a monomial and an exponential while the distant field is described by a monomial. The return currents are described with vector cylindrical harmonics and their adequate choice is guided by the topology of the currents to be described. Vector field formalism can lead to very complex models by simply adding more and more contributions, but the simpler the construction, the more efficient the model will be. The tail model resisted our efforts and was borrowed from the Tsyganenko and Usmanov (1982) model. It is not warped and is the single non-poloidal part of this model presently. This model did not use a prescribed magnetopause nor the mathematical technique employed elsewhere to satisfy the boundary conditions. As a consequence the obtention of a series of models with no field line escape in the day side and without night side flaring of the field lines required several iterations and was rather cumbersome. This iteration process was only possible with a rather simple mathematical model. The lack of data over the dipole poles near the magnetopause lead to a field line shape somehow different from the Sibeck magnetopause model. Incorporating our previous model of the ring current enabled a good description of this region in accordance with the experimental results of Sugiura and Poros (1973).

Using different data sets several series of coefficients have been derived for different $\mathrm{Kp}$. In this respect our model belongs to the series of the earlier Tsyganenko models, 1982 to 1989 (Tsyganenko and Usmanov 1982; Tsyganenko, 1987, 1989). However some problems encountered in these models have been corrected, in 
particular a good description of the near-Earth magnetic field has been obtained. Our model does not include field aligned currents nor a warped tail as in the most recent model of Tsyganenko (Tsyganenko, 1996). Its simplicity and its ease of use makes it particularly suited for a first approach in the interpretation of particle data or intensive type calculations.

Acknowledgements. We gratefully acknowledge NSSDC for making available the magnetic field measurements data base and D. H. Fairfield, N. A. Tsyganenko, A. V. Usmanov and M. V. Malkov for this achievement. We wish to thank the referee for a careful reading of the manuscript and valuable suggestions in the presentation of the mathematics.

Topical Editor K.-H. Glassmeier thanks a referee for his help in evaluating this paper.

\section{References}

Alekseev J. I., and V. P. Shabansky, A model of a magnetic field in the geomagnetosphere, Planet. Space Sci., 20, 117-133, 1972.

Choe J. Y., and D. B. Beard, The compressed geomagnetic field as a function of dipole tilt, Planet. Space Sci., 22, 595-608, 1974.

Fairfield D. H., N. A. Tsyganenko, A. V. Usmanov and M. V. Malkov, A large magnetospheric magnetic field data base, J. Geophys. Res., 99, 11319-11326, 1994.

Hilmer R. V., and G. H. Voigt, A magnetospheric magnetic field model with flexible current systems driven by independent physical parameters, 100, 5613-5626, 1995.

Kosik J. C., Quantitative magnetic field model including magnetospheric ring current, J. Geophys. Res., 94, 12021-12026, 1989.

Mead G., and D. H. Fairfield, A quantitative magnetospheric model derived from spacecraft magnetometer data, J. Geophys. Res., 80, 523-534, 1975.

Olson W. P., and K. A. Pfitzer, A quantitative model for the magnetospheric magnetic field, J. Geophys. Rev., 79, 3739-3748, 1974.
Schulz M., and M. McNab, Source surface model of the magnetosphere, Geophys. Res. Lett. 14, 182, 1987.

Schulz M., and McNab, Source surface modeling of planetary magnetospheres, J. Geophys. Res., 101, 5095-5118, 1996.

Sibeck D. G., R. E. Lopez, and E. C. Roelof, Solar wind control of the magnetopause shape, location and motion, J. Geophys. Res., 96, 5489, 1991 .

Sozou C., and D. W. Windle, Non linear symmetric inflation of a magnetic dipole, Planet. Space Sci., 17, 999-1008, 1969.

Stern D. P., Representation of magnetic fields in space, Rev. Geophys., 14, 199-214, 1976.

Stern D. P., Parabolic harmonics in magnetospheric modeling: the main dipole and the ring current, J. Geophys. Res., 90, 1085110863, 1985.

Sugiura M., and D. J. Poros, A magnetospheric field model incorporating the OGO 3 and 5 magnetic field observations, Planet. Space Sci., 1763-1773, 1973.

Tsyganenko N. A., Global quantitative models of the geomagnetic field in the cislunar magnetosphere for different disturbance levels, Planet. Space Sci., 35, 1347-1358, 1987.

Tsyganenko N. A., A magnetospheric magnetic field model with a warped tail current sheet, Planet. Space Sci., 37, 5-20, 1989.

Tsyganenko N. A., Modeling the Earth's magnetospheric magnetic field confined within a realistic magnetopause, J. Geophys. Res., 100, 5599-5612, 1995.

Tsyganenko N. A., Effects of the solar wind conditions on the global magnetospheric configuration as deduced from data based field models, in Proc. ICS-3 Conference on substorms (Versailles, France, May 12-17, 1996), ESA SP-389, pp. 181-185, Oct.1996.

Tsyganenko N. A., and A. V. Usmanov, Determination of the magnetospheric current system parameters and development of experimental geomagnetic field models based on data from IMP and HEOS satellites, Planet. Space Sci., 30, 985-998, 1982.

Voigt G. H., A three dimensional analytical magnetospheric model with defined magnetopause, Z. Geophys., 38, 319-346, 1972.

Wolf-Gladrow D. A., An introduction to poloidal and toroidal fields, Mitt; 54, Inst. Geophys. Meteorol. Univ. Koln. 1987. 